Z. klin. Chem. u. klin. Biochem.

9. Jg., S. $431-437$, September 1971

\title{
Zur Wirkung von Divicin in menschlichen Erythrocyten ${ }^{1}$ )
}

\author{
Von L. Floht' ${ }^{2}$ ), G. NIEBCH ${ }^{3}$ ) und H. REIBER \\ Aus dem physiol. chem. Institut (Direktor Prof. Dr. Dr. G. Weitzel) der Universität Tübingen
}

(Eingegangen am 24. April 1971)

\begin{abstract}
Divicin und Dialursäure bewirken auch in normalen menschlichen Erythrocyten bei entsprechend hohen Konzentrationen unter aeroben Bedingungen in vitro einen schnellen GSH-Abfall und eine Bildung von Lipidperoxiden. Zeitlich verzögert wird ein erhöhter $\mathrm{K}^{+}-\mathrm{Ver}-$ lust meßbar. Eine biologische Regeneration von GSH nach Divicin- und Dialursäurebehandlung wird nicht beobachtet, während nach Behandlung mit Diamid (Azodicarbonsäure-bis [N,N-dimethylamid]) eine partielle Reduktion des gebildeten GSSG gefunden wird. Vorherige Oxydation des intraerythrocytären GSH durch Diamid zeigt keinen signifikanten Einfluß auf das Ausmaß der Lipidperoxidbildung. Die Phänomene werden auf eine radikalisch vetlaufende Autoxydation der 0 -Dihydroxypyrimidinderivate zurückgeführt. Entsprechend zeigen Vicin und 2,4-Diamino-6-hydroxypyrimidin die charakteristischen Effekte nicht. Allerdings kann Divicin aus Vicin im Serum freigesetzt werden, wodurch ein im Vergleich zu Divicin schwacher $\mathrm{K}^{+-V e r l u s t ~ z u ~ e r k l a ̈ r e n ~ w a ̈ r e . ~ D i e ~ d u r c h ~ D i v i c i n ~ a u s g e l o ̈ s t e n ~ V e r-~}$ änderungen am Erythrocyten werden im Hinblick auf ihre mögliche Bedeutung bei der Pathogenese des Favismus diskutiert.
\end{abstract}

\section{The effect of divicine on buman erytbrocytes}

Under aerobic conditions in vitro, high concentrations of divicine and dialuric acid produce a rapid fall in GSH and the formation of lipid peroxides even in normal human erythrocytes. After a considerable delay an increased $\mathrm{K}+$ loss is observed. A biological regeneration of GSH after treatment with divicine and dialuric acid is not found, whereas GSSG formed after treatment with diamide (N,N, $N^{\prime}, N^{\prime}$ Tetramethylazoformamid) is partially reduced. A previous oxidation of intracellular GSH by diamide does not cause a significant effect on the degree of lipid peroxidation. The phenomena can be explained by the generation of free radicals during the autoxidation of the o-dihydroxy pyrimidine derivatives. Correspondingly, the characteristic effects are not seen with vicine and 2,4-diamino-6-hydroxy pyrimidine. Vicine, however, is hydrolyzed to divicine in the presence of serum. The comparably low $\mathrm{K}+$ loss observed with vicine may be due to the formation of divicine during the incubation. The alterations of the erythrocytes conditioned by divicine are discussed with respect to their possible role in the pathogenesis of favism.

Seit 1956 ist bekannt, daß ein genetischer Mangel an Glucose-6-phosphatdehydrogenase in den toten Blutzellen mit einer erhöhten Bereitschaft zu hämolytischen Krisen einhergeht (1). Die Empfindlichkeit der enzymopenischen Erythrocyten gegen Inhaltsstoffe der Vicia faba haben dem Krankheitsbild den Namen Favismus eingeträgen. Trotz zahlreicher Untersuchungen jedoch ist über den pathogenetischen Mechanismus der hämolytischen Krise noch kein abschließendes Urteil möglich $(2,3)$. Als toxische Komponenten der Fava-Bohnen konnten die Pyrimidinderivate Divicin und Isouramil identifiziert werden, die in der Bohne überwiegend als in vitro unwirksame Glucoside vorliegen $(4,5)$.

Divicin und Isouramil bewirken ähnlich wie Phenylhydrazine und Hydroxylamine eine Zerstörung des intraerythrocytären Glutathions $(4,5)$. Die gesteigerte Anfälligkeit von Glucose-6-phosphatdehydrogenaseMangel-Zellen wird auf die verminderte Reduktionskapazität für Glutathion zurückgeführt (2). Diese Hypothese wird durch die Beobachtung gestützt, daß bei GSH=Mangel-Zuständen, die nịcht durch einen Glucose-6-phosphatdehydrogenase-Mangel bedingt sind,

1) Mit Unterstützung der Deutschen Forschungsgemeinschaft.

2) Physiol. chem. Institut der Universität Tübingen, 74 Tübingen, Hoppe-Seyler-Str. 1.

3) z. Z. Institut f. physiol. Chemie, 355 Marburg/Lahn.

4) z. Z. Institut f. biophysikalische Chemie, 34 Göttingen. ebenfalls eine erhöhte Hämolysebereitschaft festgestellt werden kann (6-Phosphogluconatdehydrogenase-Mangel $(6,7)$, Glutathionsreduktase-Mangel $(8,9)$, Synthetase-Mangel $(9,10,11))$. Demgegenüber zeigten Kosower und Mitarbeiter $(12,13)$, daß eine quantitative Oxydation des intraerythrocytären Glutathions zumindest kurzfristig keine nachteiligen Folgen für den Erythrocyten hat, sofern zusätzliche oxydative Noxen ausgeschlossen werden können.

$\mathrm{Da}$ viele hämolysierende Reagenzien im Erythrocyten teils spontan, teils unter katalytischer Beteiligung von Hämoglobin $\mathrm{H}_{2} \mathrm{O}_{2}$ freisetzen (14), liegt es nahe, in der Glutathionperoxydasereaktion die entscheidende Funktion des erythrocytären Glutathions zu suchen (3). Neben einer Akkumulation von $\mathrm{H}_{2} \mathrm{O}_{2}$ könnte jedoch auch eine Bildung von Lipidperoxiden in der Etythrocytenmembran zu einer Hämolyse führen (15-19). Eine parallele Zerstörung des intrazellularen Glutathions müßte die deletäre Wirkung der Lipidperoxide potenzieren, da letztere in diesem Fall nicht mehr via GSH-Peroxydase abgebaut werden könnten.

Vorliegende Arbeit berichtet über die Wirkungen von Divicin und verwandten Verbindungen am gesunden Humanerythrocyten. Es sollte geklärt werden, welchen strukturellen Parametern des Divicins die toxische Wirkung zuzuschreiben ist. Darüber hinaus wurden die Effekte auf den GSH-Spiegel, die ungesättigten Membranlipide und die Kaliumretention der Erythro- 
cyten zeitabhängig verfolgt, um weitere Einblicke in den Mechanismus der Divicin-bedingten Zellschädigung zu erhalten.

\section{Material und Methoden}

Chemikalien

2-Thiobarbitursäure-Hydrat p. a. wurde von EGA-Chemie, Steinheim, 3,3'-Dithio-bis[nitrobenzoesäure], Dialursäure und Malonaldehydtetraacetal von Schuchardt, München, Lewatit $S 100$ von Bayer, Leverkusen, und alle weiteren Reagenzien von Metck AG, Darmstadt, bezogen.

\section{Präparate}

I Vicin: Vicin wurde modifiziert nach GMExIN und HasenMarer (20) wie folgt isoliert: $500 \mathrm{~g}$ Samen von Vicia sativa (Württembergische Landwirtschaftliche Zentralgenossenschaft) werden $2 \mathrm{mal}$ mit warmem Leitungswasser gewaschen und über Nacht mit 21 Leitungswasser stehengelassen. Die vorgekeimten Samen werden in einem Fleischwolf zerkleinert und in 21 Wasser resuspendiert, mit 21 Methanol versetzt und am Rückfluß gekocht. Nach Zentrifugation wird der Niederschlag erneut mit 21 Wasser und 21 Methanol extrahiert. Die vereinigten Uberstände werden im Vakuum bei $40^{\circ}$ vom Methanol befreit, mit Wasser auf das ursprüngliche Volumen verdünnt und auf eine Lewatit S 100Säule ( $\mathrm{H}^{+}$-Form; Bettrolumen $250 \mathrm{ml}$ ) aufgetragen. Die Säule wird mit 2 Vol. Wasser gewaschen. Vicin wird mit 2proz. $\mathrm{NH}_{3}-$ Lösung eluiert, das Eluat lyophilisiert und in heißem Wasser gelöst. Beim Erkalten kristallisiert Vicin in farblosen Nadeln aus. Reinigung effolgt durch zweimaliges Umkristallisieren aus heißem Wasser.

Fp.: $243^{\circ}$; Ausbeute: $383 \mathrm{mg}$

$\mathrm{C}_{10} \mathrm{H}_{16} \mathrm{O}_{7} \mathrm{~N}_{4} \cdot \mathrm{H}_{2} \mathrm{O}(322,2)$

Ber.: C 37,25 H 5,63 N 17,39

Gef.: C 37,33 H 5,77 N 17,20

II Divicin aus Vicin (21): $50 \mathrm{mg}$ Vicin in $0,5 \mathrm{ml} 2 \mathrm{~N} \mathrm{H}_{2} \mathrm{SO}_{4}$ werden im Einschlußrohr unter $\mathrm{N}_{2} 15 \mathrm{Min}$. auf $100^{\circ}$ erhitzt. Nach Zugabe von $1,25 \mathrm{ml}$ Äthanol fällt Divicin als Sulfat nahezu quantitativ aus.

Ausbeute: $14,3 \mathrm{mg}$ ( $45 \%$ d. Th.)

$\mathrm{C}_{4} \mathrm{O}_{2} \mathrm{~N}_{4} \mathrm{H}_{6} \cdot 1 / 2 \mathrm{H}_{2} \mathrm{SO}_{4}(191,2)$

Ber.: C 25,10 H 3,70

Gef.: C 24,60 H 3,83.

III 2,4-Diamino-6-bydroxy'pyrimidin-sulfat wurde nach 1. c. (22) dargestellt.

IV 2,4-Diamino-5,6-dibydroxypyrimidin-bydrocblorid (Divicin) wurde aus III über den 5-Schwefelsäure-Ester des Divicins analog 1. c. (23) synthetisiert. Analysenwerte und biologische Wirkungen entsprachen denen des aus Vicin dargestellten Produktes.

$\mathrm{V}$ Hydrazodicarbonsäure-bis[ $N, N$-dimetbylamid] wurde nach 1 . c. (24) dargestellt.

VI Azodicarbonsäure-bis[ $N, N$-dimetbylamid] („Diamid“) wurde aus $\mathrm{V}$ nach 1. c. (24) gewonnen.

\section{Inkubationsversuche}

Heparinisiertes Humanblut wurde $10 \mathrm{Min}$. bei $1400 \mathrm{~g}$ und $2^{\circ}$ zentrifugiert. Serum und Leukocytenschicht wurden verworfen. Die Erythrocyten wurden $1 \mathrm{mal}$ in isotonischem Glucose-haltigem Medium (I) suspendiert, zentrifugiert und nach Abziehen des Uberstandes mit Glucose-freiem Medium (II) auf einen Hämatokrit von 40 eingestellt. Die Medien hatten folgende Zusammensetzung: Glucose-haltiges Medium (I): $0,01 \mathrm{M} \mathrm{NaH} \mathrm{NO}_{4} / \mathrm{Na}_{2} \mathrm{HPO}_{4}$, $\mathrm{pH} 7,35 ; 0,004 \mathrm{M} \mathrm{KCl} ; 0,126 \mathrm{M} \mathrm{NaCl} ; 0,005 \mathrm{M}$ Glucose. Glucosefreies Medium (II): $0,01 \mathrm{M} \mathrm{NaH} \mathrm{NaO}_{4} / \mathrm{Na}_{2} \mathrm{HPO}_{4}, \mathrm{pH} 7,35 ; 0,004 \mathrm{M}$ $\mathrm{KCl} ; 0,129 \mathrm{M} \mathrm{NaCl}$. In beiden Fällen betrug die Gefrierpunktserniedrigung $0,53^{\circ}$.

Die Inkubationen wurden bei einem Hämatokrit von 20 bei $37^{\circ}$ durchgeführt. Hierzu wurden jeweils 2 Teile Erythrocytensuspen- sion (Hämatokrit 40) mit 1 Teil Medium II oder „Diamid“c in Medium II versetzt und nach einer Vorinkubation von $30 \mathrm{Min}$. mit 1 Teil einer toxischen Substanz in Medium II versetzt. Zur Beobachtung spontaner Veränderungen der Erythrocyten wurden nur 2 Teile Medium II zugegeben. Die Suspensionen wurden mit einer Frequenz von 140/Min. unter Luftzutritt geschüttelt. Zu gegebenen Zeiten wurden Proben zur Bestimmung des GSHGehaltes, des $\mathrm{K}^{+}$-Verlustes sowie der Akkumulation von Lipidperoxiden entnommen.

\section{Analytische Verfahren}

Kaliumverlust: Nach Zentrifugation einer Suspensionsprobe bei $800 \mathrm{~g}$ wurden $200 \mu \mathrm{l}$ des Uberstandes mit Wasser auf $5 \mathrm{ml}$ aufgefüllt und der $\mathrm{K}^{+}-$Gehalt flammenspektroskopisch bestimmt (Grundgerät PMQ II von Zeiss; Monochromator M4Q II; Brenner für Acetylen-Luft-Gemisch; Kalium-Hohlkathodenlampe, $\lambda_{\max }=766,5 \mathrm{~nm}$; automatische Registrierung).

Glutathionbestimmung: erfolgte mit EllmaN's Reagenz nach der von BEUTLER und Mitarbeitern (25) ausgearbeiteten Methode.

Bestimmung der Lipidperoxide: Es wurde die Thiobarbitursäuremethode (26) verwendet. Für unsere $Z$ wecke erwies sich folgende Modifikation als optimal: $200 \mu \mathrm{l}$ Erythrocytensuspension (Hämatokrit 20) wurden zu $5 \mathrm{ml}$,Thiobarbitursäure-Reagenzlösung“ gegeben. (Die Thiobarbitursäure-Reagenzlösung wurde wie folgt bereitet: Die für eine 0,5proz. Thiobarbitursäurelösung berechnete Menge Thiobarbitursäure wird in $80 \%$ des vorgesehenen $\mathrm{H}_{2} \mathrm{O}$ heiß gelöst, mit der gewünschten Menge Trịchloressigsäure versetzt (Endkonzentration 10\%) und auf $100 \%$ mit Wasser aufgefüllt. Beim Erkalten fällt Thiobarbitursäure partiell wieder aus und muß vor Gebrauch abfiltriert werden.) Die Reaktionslösung wurde im Ölbad bei $85^{\circ}$ gehalten. Nach exakt $40 \mathrm{Min}$. wurde die Farbstoffbildung durch Uberführen ins Eisbad beendet. Nach Zentrifugation wurde die Extinktion bei $532 \mathrm{~nm}$ gemessen und durch Subtraktion der unspezifischen Extinktion bei $600 \mathrm{~nm}$ korrigiert.

Zur Beurteilung der Ergebnisse deș Thiobarbitursäure-Testes seien folgende Bemerkungen vorweggeschickt: 1. Während mit Malonaldehyd als Eichsubstanz unter den verwendeten Testbedingungen die Farbstoff bildung nach etwa $20 \mathrm{Min}$. abgeschlossen ist, wird mit der Érythrocytensuspension ein kontinuierlicher Extinktionsanstieg beobachtet, der auch nach $90 \mathrm{Min}$. noch keinen definierten Endwert erreicht. Es verbieten sich daher Absolutangaben über die Menge an Lipidperoxiden in biologischem Material (vgl. Ref. 27, 28). 2. Bei der Bestimmung von Thiobarbitursäure-reaktivem Material in Erythrocytensuspensionen werden stark streuende Ergebnisse erhalten, so daß bei Doppeibestimmungen nur Abweichungen gegenüber den Kontrollen von mehr als $25 \%$ als signifikant betrachtet werden dürfen $(p=0,05)$.

\section{Ergebnisse}

\section{Einfluß von Divicin und verwandten Verbindungen auf den GSH-Spiegel von Erytbrocyten}

In Übereinstimmung mit älteren Befunden $(4,5)$ wurde durch Behandlung von normalen menschlichen Erythrocyten mit Divicin (2,4-Diamino-5,6-dihydroxypyrimidin) und der strukturverwandten Dialursäure (2,4,5,6-Tetrahydroxypyrimidin) in relativ hoher Konzentration ein schneller Abfall des intraerythrocytären Glutathions beobachtet (vgl. Abb. 1 und 2). Vicin, das $\beta$-Glucopyranosid des Divicins, und 2,4-Diamino-6hydroxypyrimidin zeigten keinen Einfluß auf den GSH-Spiegel.

Vergleicht man den zeitlichen Verlauf der intrazellulären GSH-Konzentration unter Divicineinwirkung 


\section{PLASTIBRAND-Wasserstrahlpumpe}

Erzeugnis einer Fabrik für Vakuumpumpen

Hohe Saugleistung; Rückschlagsicherheit auch bei niedrigem Wasserdruck

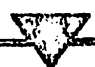

Keine Wassereinbrüche in die Apparatur beim Abstellen der Pumpe

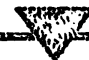

Einfach zu montieren

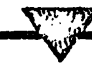

Lange Lebensdauer
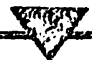

Geringe Betriebskosten

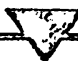

BRAND-GERÄTE WEIL DIE

RENTABILITÄT

ENTSCHEIDET
Die inneren Abmessungen einer Wasserstrahlpumpe kann man "ausprobieren" oder errechnen.

Die Plastibrand-Pumpe ist errechnet

Eingebautes

Rückschlagventil

Gewindeanschlüsse $1 / 2$ ", $3 / 8^{\prime \prime}, 3 / 4 ", M 22 \times 1$ oder Schlauchtülle

Einfacher Aufbau aus wenigen, robusten

Polypropylenteilen

Niedriger

Wasserverbrauch durch sorgfältig abgestimmte Querschnittsverhältnisse der Düsen

\section{Günstiger}

Anschaffungspreis

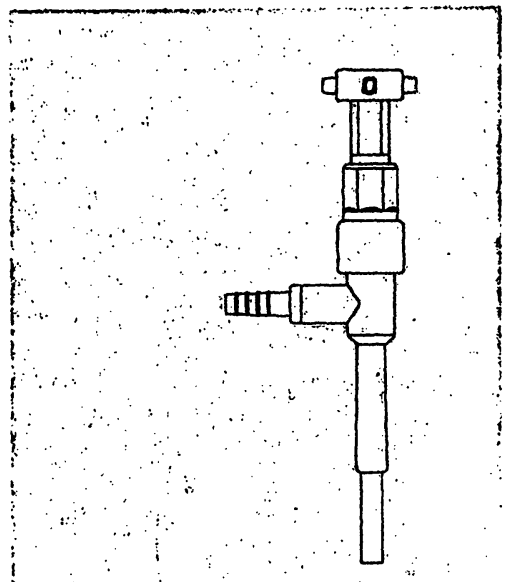

Mit Gewindeanschlub

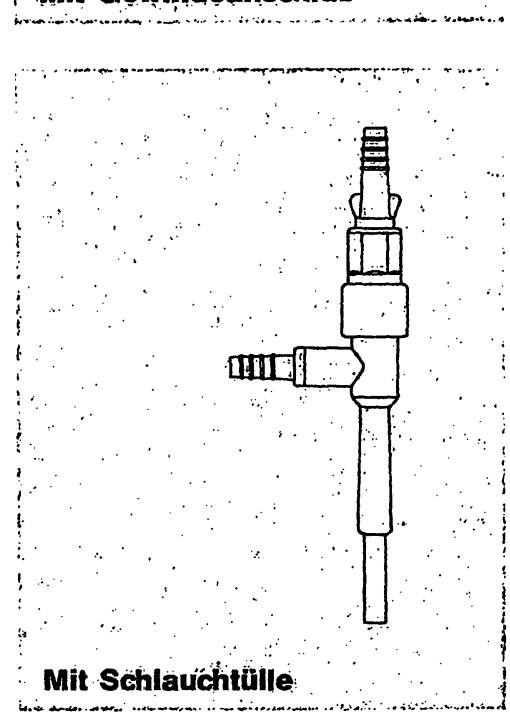



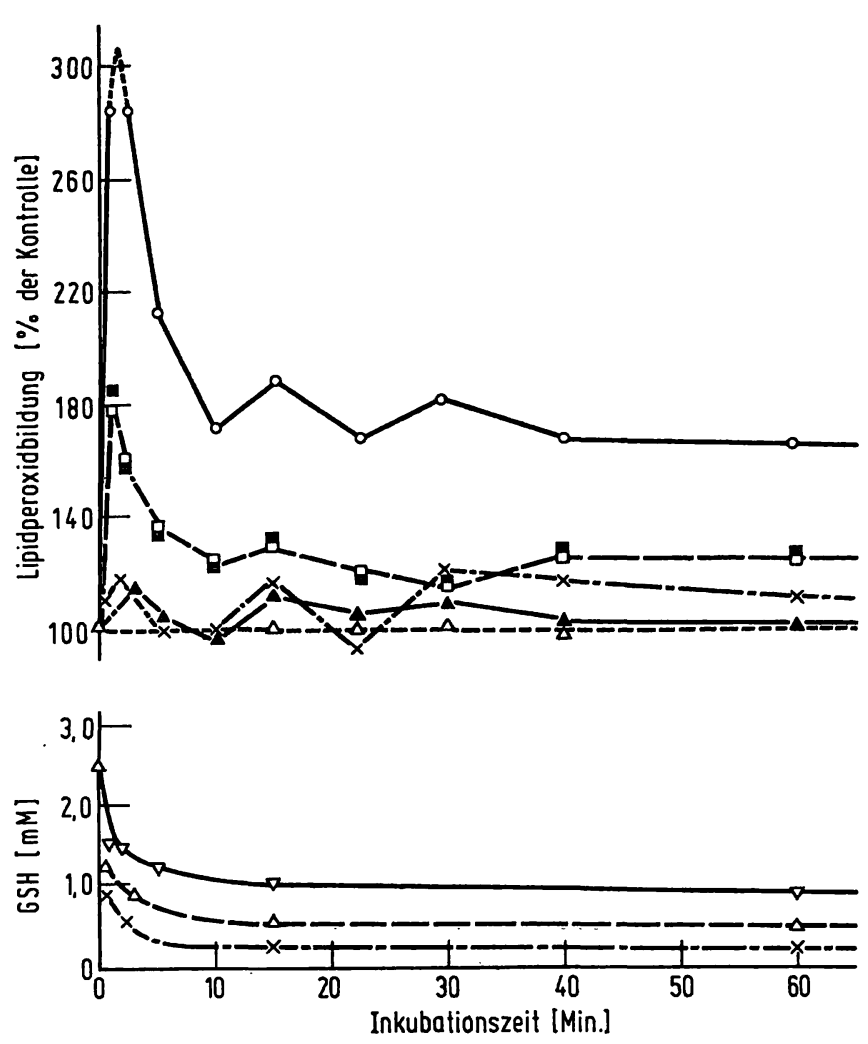

Abb. 1

Lipidperoxidbildung und GSH-Abfall in einer Erythrocytensuspension (Hamatokrit: 20) bei Einwirkung von Divicin in verschiedenen Konzentrationen. Die Inhubationsbedingungen sind unter ,Methoden" beschrieben. Die Lipidperoxidwerte (obere Skala) sind in \% de Leerwertes $\left(=100 \% ; \mathrm{E}_{\mathrm{bag}}=0,15\right)$ angegeben. Die GSH-Molarität (untere Skala) ist auf das Volumen sedimentierter Zellen bezogen. Die ausgefüllten Symbole kennzeichnen Versuche, bei denen vor Divicinzugabe GSH durch Diamid $(0,54 \mathrm{~mm})$ oxydiert wurde. Divicin wurde unter $N_{2}$ gelöst, bei der höchsten Konzentration (10 mM) wurde Divicin als Suspension zugegeben. Die Divicin-Konzentrationen waren wie folgt:

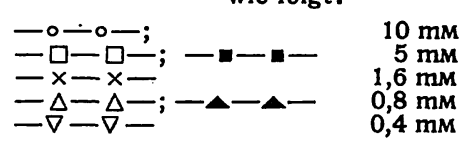

(Abb. 1) mit entsprechenden Kurven nach Diamidbehandlung, so fällt ein entscheidender Unterschied auf: Bei Behandlung von Ërythrocyten mit einer Diamidkonzentration $(0,54 \mathrm{~mm})$, die der zweifachen Menge des intraerythrocytären Glutathions äquivalent ist, erhalten wir eine quantitative GSH-Oxydation. Da die verwendeten Erythrocyten jedoch Glucose enthalten (vgl. Methoden) beginnt nach etwa $10 \mathrm{Min}$. eine Rückbildung reduzierten Glutathions bis $\mathrm{zu}$ etwa $50 \%$ des Ausgangswertes. Bei geringerer Diamidkonzentration kann eine nahezu quantitative Regeneration des reduzierten Glutathions erzielt werden. Ein durch Glucosemangel bedingter GSH-Abfall wird unter den gewählten Bedingungen bei Diamid-behandelten und unvorbehandelten Erythrocyten nicht vor der fünften Inkubationsstunde beobachtet. Demgegenüber bleibt die GSH-Konzentration bei Divicineinwirkung nach $10 \mathrm{Min}$. annähernd konstant, selbst wenn die eingesetzte Divicinkonzentration nur zu einem 50 prozentigen GSH-Abfall ausreichte. Ferner wurde beobachtet, daß die Oxydation von GSH durch Divicin keiner übersichtlichen Stöchiometrie entspricht:
Mit sinkender Divicinkonzentration nimmt die relative Wirksamkeit von Divicin rasch zu (vgl. Tab. 1).

Tab. 1

Stöchiometrie der GSH-Oxydation durch Divicin in einer Erythrocytensuspension

\begin{tabular}{ccc}
\hline Divicin (mM) & GSH (mM) & $\begin{array}{c}\text { Mol ,oxydiertes“" Glutathion } \\
\text { prc Mol Divicin }\end{array}$ \\
\hline 0 & 0,86 & - \\
1 & 0,20 & 0,66 \\
0,5 & 0,37 & 0,98 \\
0,1 & 0,70 & 1,60 \\
\hline
\end{tabular}

\section{Bildung von Lipidperoxiden}

Um bei normalen menschlichen Erythrocyten eine Lipidperoxidbildung durch Dialursäure beobachten zu können, müssen extrem hohe Konzentrationen eingesetzt werden ( $\mathrm{Abb}, 2$ ). $\mathrm{Da}$ wir primär an der Frage
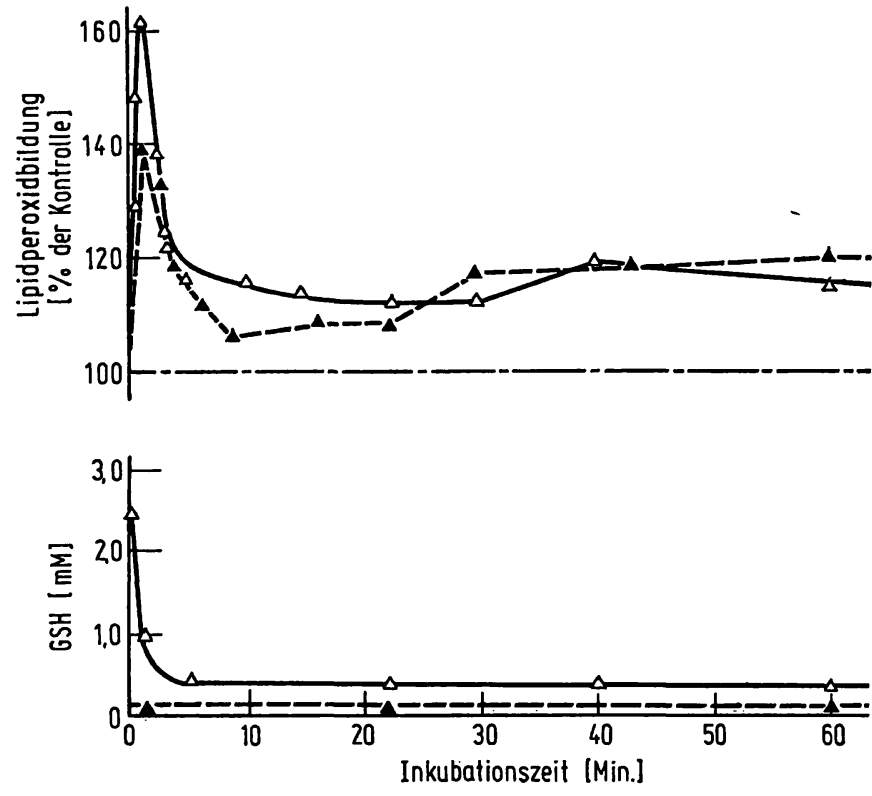

Abb. 2

Lipidperoxidbildung und GSH-Abfall in einer Erythrocytensuspension bei Einwirkung von $10 \mathrm{~mm}$ Dialursäure - -1 nach Vorbehandlung mit Diamid; $-\Delta-\Delta-$ unvorbehandelt.

Weitere Einzelheiten vergleiche Legende zu Abbildung

interessiert waren, ob überhaupt von Divicin und Strukturanalogen dieser Reaktionsweg beschritten wird, nahmen wir in unseren Versuchen hohe Konzentrationen in Kauf, obwohl mit einem derartigen Blutspiegel bei Intoxikationen nicht $\mathrm{zu}$ rechnen ist. Wie aus Abbildung $1 \mathrm{zu}$ ersehen ist, werden ab $5 \mathrm{~mm}$ Divicin signifikante Mengen Lipidperoxide erfaßbar. Qualitativ ähnliche Verhältnisse werden mit Dialursäure beobachtet, während 2,4-Diamino-6-hydroxypyrimidin selbst bei $10 \mathrm{~mm}$ wirkungslos bleibt. Vicin, das wie erwähnt keinen Einfluß auf den GSH-Spiegel zeigt, bewirkt ebenfalls in hohen Konzentrationen (10 mM) keine signifikante Lipidperoxidbildung. (Allerdings werden mit hohen Vicin-Konzentrationen stets erhöhte Extinktionen beim Thiobarbitursäure-Test ermittelt. Diese 
zeigen aber beim Inkubationsversuch nicht die typische Zeitabhängigkeit und werden auch beobachtet, sofern Vicin erst nach Fällung der Zellsuspension mit Trichloressigsäure zugegeben wird. Möglicherrweise wird demnach unter den Bedingungen des Thiobarbitursäure-Testes Divicin aus Vicin freigesetzt, was zu einer begrenzten Bildung von Malonaldehyd unter den Bedingungen der Farbstoffentwicklung führt.) Die Bildung von Lipidperoxiden bei physiologischem $\mathrm{pH}$ ist also wie die Fähigkeit zur irreversiblen Glutathionoxydation an eine freie o-Dihydroxy-Gruppierung der Pyrimidinderivate gebunden.

Eine mit Serum unter $\mathrm{O}_{2}$-Ausschluß vorinkubierte Vicinlösung bewirkte eine signifikante Lipidperoxidbildung in der sauerstoffhaltigen Erythrocytensuspension, während bei einer direkten Inkubation von Vicin mit serumhaltigen Erythrocyten unter $\mathrm{O}_{2}$ die Signifikanzgrenze nicht erreicht wurde (Abb. 3). Divicin

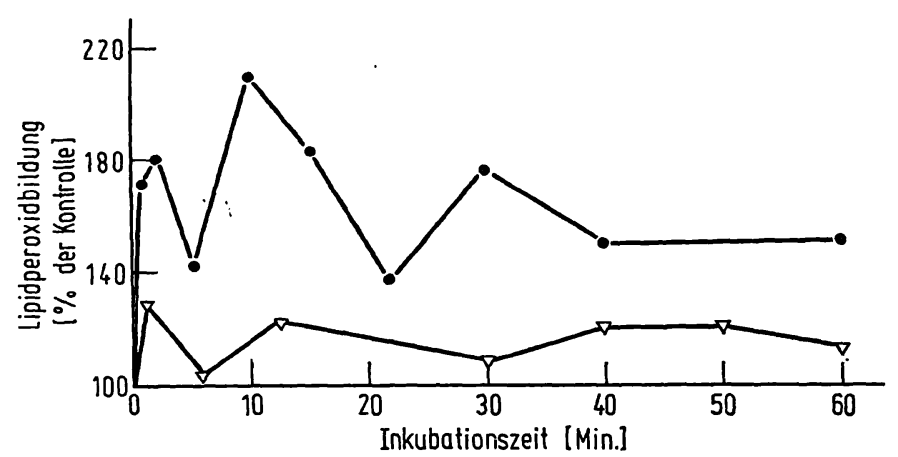

Abb. 3

Lipidperoxidbildung durch Vicin in einer Erythrocytensuspension

- - - - Vicin wurde mit Serum $30 \mathrm{Min}$. bei $37^{\circ}$ unter $\mathrm{N}_{2}$ vorinkubiert, anschließend mit der $\mathrm{CO}_{2}$-haltigen Erythrocytensuspension inkubiert. "Vicin-Konzentration" im Glykosid); Hämatokrit 20

$-\nabla-\nabla-$ : Entsprechender Versuch, doch wurde Vicin nicht unter $\mathrm{N}_{2}$ vorinkubiert, sondern unmittelbar zum Inkubat gegeben. Eine signifikante Erhöhung der Peroxidwerte wird unter diesen Bedingungen nur in einem einzelnen Meßpunkt erreicht. Die Lipidperoxide (thiobarbitursäure-positives Material) sind in \% des Leerwertes
(ohne Vicin $=100 \%$ ) angegeben

wird demnach aus Vicin durch unspezifische Glykosidasen des Serums freigesetzt. Wird jedoch das Glucosid unter aeroben Bedingungen hydrolysiert, so kann Divicin nicht in hinreichender Menge akkumulieren, um eine meßbare Peroxidkonzentration zu erzielen.

Verfolgt man den Lipidperoxidgehalt unter Dialursäure-Einwirkung über die Zeit, so wird offenbar, daß die Bildung von einem schnellen Abbau überlagert ist (Abb. 1 und 2). Der in unseren Versuchen beobachtbare steile Abfall der Lipidperoxide nach der initialen Bildung ist jedoch unmöglich durch eine enzymatische GSH-abhängige Reduktion bedingt, da zum Zeitpunkt des Abfalls bereits kein GSH mehr im Erythrocyten vorhanden ist. In einzelnen Versuchsansätzen wurde vor der Inkubation der Erythrocyten mit Divicin bzw. Dialursäure Glutathion quantitativ durch Diamid oxydiert. Sofern eine nennenswerte Reduktion von Lipidperoxiden durch GSH-Peroxydase an den Ab- bauvorgängen beteiligt sein sollte, wäre in den Diamidbehandelten Erythrocyten eine erhöhte initiale NettoBildung von Peroxiden zu fordern. Die Ergebnisse aus Abbildung 1 und 2 entsprechen jedoch nicht diesen Erwartungen. Lediglich bei einer Divicinkonzentration von $0,8 \mathrm{~mm}$ liegen die Peroxidwerte in Diamid-behandelten Exythrocyten geringfügig höher; doch sind die Differenzen nicht als statistisch signifikant $z u$ betrachten. Ebensowenig konnte eine Abhängigkeit der spontanen Lipidperoxidbildung vom GSH-Gehalt der Zellen gezeigt werden. Durch einen Parallelversuch wurde ausgeschlossen, daß entstehendes Hydrazodicarbonsäure-bis[N,N-dimethylamid] eine eventuelle Lipidperoxidbildung beeinträchtigt.

\section{$K^{+}-$Verlust der Erytbrocyten}

Als Indikator für eine Membranschädigung der Erythrocyten betrachteten wir die Abgabe von $\mathrm{K}^{+}$-Ionen an das Inkubationsmedium. Bei kurzen Inkubationszeiten beobachteten wir selbst bei hohen Konzentrationen der toxischen Substanzen keine signifikanten Unterschiede zur Kontrolle. Erst bei Langzeitinkubationen wurden mit allen Verbindungen, die intrazellulär Glutathion oxydieren, ein deutlich erhöhter $\mathrm{K}^{+}$-Verlust und eine Verkürzung der in-vitro-Lebenszeit der Erythrocyten sichtbar.

Von den Pyrimidinderivaten bewirkte 2,4-Diamino-6hydroxypyrimidin $(10 \mathrm{~mm}$ ) keinerlei Funktionsstörungen der Erythrocytenmembran. Mit Vicin hingegen konnten von der zwanzigsten Inkubationsstunde an signifikante Membranschädigungen beobachtet werden. Eine Hydrolyse des Vicins konnte in Anbetracht der langen Inkubationszeiten jedoch nicht ausgeschlossen werden.

Relativ frühzeitig stieg der $\mathrm{K}^{+}-$Verlust bei Behandlung der Erythrocyten mit Dialursäure oder Divicin. Signifikante Unterschiede zur Kontrolle konnten bereits

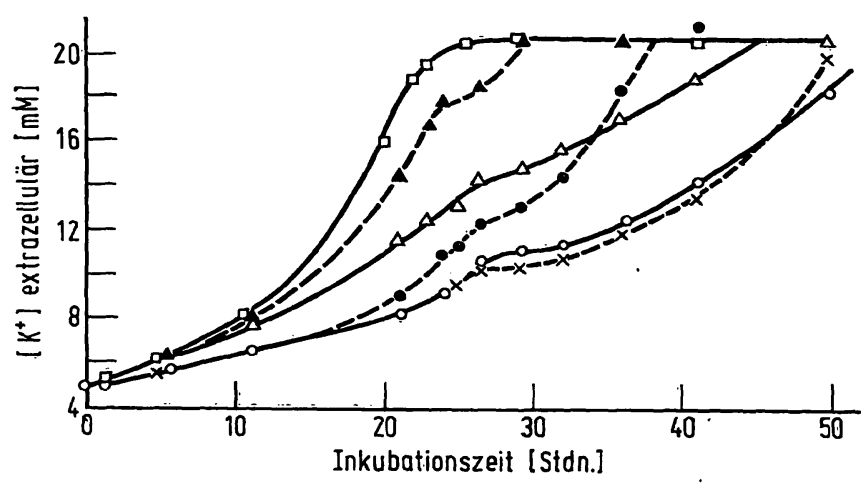

Abb. 4

$\mathrm{K}^{+-}$-Verlust normaler Erythrocyten unter Einwirkung von 二口- $\square-$ Dialsäure, 二 $\triangle-\Delta-$ Diamid, 二-x- $\times$ - Vicin 二 $x-x-$ 2,4-Diamino-6-hydroxypyrimidin,

Vorbehandlung und Inkubationsbedingungen siehe Methoden. Aufgetragen ist das extrazelluläre $\mathrm{K}^{+}$in mM gegen die Zeit in Stunden.
Die Konzentration der toxischen Reagenzien betrug $10 \mathrm{~mm}$ mit

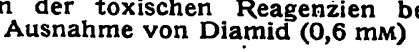


nach wenigen Stunden beobachtet werden (Abb. 4), und nach spätestens 30 Stunden war die maximale extrazellulärc $\mathrm{K}^{+}$-Konzentration erreicht.

\section{Diskussion}

5,6-Dihydroxypyrimidinderivate bewirken am Erythrocyten in Gegenwart von Saucrstoff folgende Veränderungen:

1. Eine schnelle irreversible GSH-Oxydation,

2. cine Bildung von Lipidperoxiden und

3. einen $\mathrm{K}^{+}$-Verlust mit nachfolgender Hämolyse.

Die hämolytische Krise des Favismus ist durch eine erhöhte Blutmauserung im reticuloendothelialen System, in schweren Fällen durch intravasale Hämolyse charakterisiert, so daß an Veränderungen der Erythrocytenmembran als auslösende Faktoren gedacht werden muß. Es soll nun diskutiert werden, welche in vitro beobachtbaren Veränderungen der Erythrocyten bei Divicin-Einwirkung pathophysiologisch relevant sein könnten.

Zunächst muß ein Angriff hämolysierender Substanzen auf SH-Gruppen der Erythrocytenmembran in Betracht gezogen werden. Bei Schwermetallvergiftung mit verkürzter Lebensdauer der Erythrocyten steht dieser Reaktionsweg offenbar im Vordergrund (29). Kürzlich wurde aber auch die Menadion-bedingte Hämolyse auf die Addition von Menadion an MembranSH-Gruppen zurückgeführt (30). Gerade in dieser Untersuchung wurde jedoch auf den Unterschied im Mechanismus von Menadion- und Dialursäure-bedingter Hämolyse hingewiesen, der sich in der divergierenden Beeinflußbarkeit durch Antioxydantien äußert. Darüber hinaus zeigen unsere Untersuchungen, daß die Funktionsstörungen der Membran erst um Stunden verzögert auftreten. Es liegt daher nahe, die primären Schädigungen der Erythrocyten durch Divicin und Dialursäure in den drastischen Veränderungen gleich zu Beginn der Inkubation zu suchen.

Die hämolytisch wirkenden Pyrimidinderivate Isouramil, Divicin und Dialursäure zeichnen sich durch extreme Instabilität gegen Sauerstoff aus. Allem Anschein nach verläuft diese Autoxydation radikalisch, wodurch einerseits die Schnelligkeit der initialen Veränderungen am Erythrocyten, andererseits die unübersichtliche Stöchiometrie der Reaktionen zwanglos erklärt werden kann. Die Folgen dieses Autoxydationsprozesses manifestieren sich sowohl im Cytosol (irreversibler GSH-Sturz) als auch an der Membran selbst (Lipidperoxidbildung).

Die Hypothese, daß ein intraerythrocytärer GSHAbfall Hämolyse auslöst, ist ebenso bestechend wie unbewiesen $(2,3,16,19)$. Da GSH via GSH-Peroxydase Hydroperoxide beliebiger Struktur, wie sie bei Autoxydationsvorgängen anfallen, reduzieren kann $(31-33)$, bietet das GSH-System zumindest im cytoplasmatischen Raum den effektivsten Oxydationsschutz. Bei gesenktem
GSH-Spiegel wäre die Zelle der deletären Wirkung der Peroxide preisgegeben.

Die vorliegenden Ergebnisse können nur begrenzt zugunsten dieser Vorstellung interpretiert werden:

1. Von allen Reagenzien, die GSH intrazellulär oxydieren, wird Diamid als das spezifischste angesehen (13). Bci Langzeitexperimenten mit Diamid wird eindeutig eine Funktionsstörung der Membran crkennbar. Da jedoch nach neueren Befunden (34) neben GSH zumindest auch Flavine und Pyridinnucleotide durch Diamid oxydiert werden, bleibt unser Befund mehrdeutig.

2. Bei Behandlung mit $0-D$ ihydroxypyrimidinen ist der GSH-Abfall diejenige Zellschädigung, die zeitlich am frühesten und bei den geringsten Konzentrationen beobachtet wird. Doch kann auch aus diesem Befund nicht zwingend abgeleitet werden, daß die GSHOxydation dic primäre Schädigung am Erythrocyten darstellt. TSEN und Collier untersuchten die Beeinflußbarkeit der Dialursäurewirkung durch Tokopherol $(18,19)$. In diesen Versuchen wurde einc wachsende Hämolyseneignung und Bildung von Lipidperoxiden mit sinkendem Tokopherolgehalt gefunden, während der GSH-Spiegel mit der Hämolyseneigung nicht korrelierbar war (19). Entsprechend diesen Befunden beobachten wir keine Steigerung der Lipidperoxidbildung nach vorheriger GSH-Oxydation mit Diamid.

3. $\mathrm{Da}$ der durch Divicin und Dialursäure bedingte GSH-Sturz ohnehin irreversibel verläuft, kann die erhöhte Empfindlichkeit von Glucose-6-phosphatdehydrogenase-Mangel-Zellen gegenüber diesen Substanzen nicht ohne Zusatzannahmen auf die erniedrigte Reduktionskapazität für Glutathion zurückgeführt werden.

Es muß daher crwogen werden, ob nicht in der Oxydation ungesättigter Membranlipide durch o-Dihydroxypyrimidine die primäre Schädigung der Erythrocyten zu sehen ist. Gegen diese Annahme können allerdings die extrem hohen Konzentrationen von Divicin und Dialursäure ins Feld geführt werden, unter denen diese Schädigung erst sichtbar wird.

Um dieses Argument zu entkräften, soll zunächst die Thiobarbitursäuremethode, die leider z. Z. die einzige Möglichkeit zur spezifischen Bestimmung von Lipidperoxiden darstellt, einer kritischen Betrachtung unterzogen werden: Obwohl die Thiobarbitursäuremethode Malonaldehyd mit hoher Empfindlichkeit nachweist, ist sie nicht gleichermaßen empfindlich zur Bestimmung von Lipidperoxiden, da die Freisetzung von Malonaldehyd aus peroxydicrten Lipiden nur ein Nebenweg innerhalb einer komplexen Reaktionsfolge darstellt (35). Dazu kommt, daß nur Peroxide von Fettsäuren mit mehr als 2 Doppelbindungen mit Thiobarbitursäure die charakteristische Färbung ergeben. Der Anteil höher ungesättigter F'ettsäuren in den Phospholipiden der Erythrocytenmembran beträgt aber nur etwa $50 \%$ allcr ungesättigten Fettsäuren (36). Die 
Erfassungsgrenze des Testes für Lipidperoxide ist nicht exakt anzugeben (vgl. 27, 28, 35), dürfte aber relativ hoch liegen.

Ein weiterer Umstand erschwert die Beurteilung der Ergebnisse: Zu gegebener Zeit sind immer nur „Momentaufnahmen" des kinetischen Gleichgewichts von Peroxidbildung und Abbau möglich. Da der Abbau offenbar recht schnell verläuft (vgl. Abb. 1 und 2), werden signifikante Steigerungen des Lipidperoxidgehaltes erst bei entsprechend hohen Bildungsraten meßtechnisch erfaßbar.

Nun zeigen aber unsere Untersuchungen, daß ein im Erythrocyten ablaufender Autoxydationsproze $\beta$ an der Zellmembran nicht spurlos vorübergeht, auch wenn sich die Peroxidwerte nach verhältnismäßig kurzer Zeit weitgehend normalisieren. Mit einer zeitlichen Verzögerung von mehreren Stunden lassen sich eindeutige Störungen der Membranfunktion messen. Diese Funktionseinbuße nach Peroxydierung der Membranlipide basiert nach ЈАСОв und Lux (16) auf einer Zerstörung von Phosphatidyläthanolamin und essentiellen Membran-SH-Gruppen.

Lipidperoxide können im Verlaufe eines Autoxidationsprozesses unmittelbar oder durch Vermittlung von $\mathrm{H}_{2} \mathrm{O}_{2}$ entstehen. $\mathrm{Da}$ die Bildung von Lipidperoxiden mit Dialursäure durch Tokopherol effektiver zu hemmen ist, als die $\mathrm{H}_{2} \mathrm{O}_{2}$-bedingte Lipidperoxydation (18), kann angenommen werden, daß bei der durch oDihydroxypyrimidine katalysierten Reaktion der Umweg über eine $\mathrm{H}_{2} \mathrm{O}_{2}$-Bildung zumindest nicht ausschließlich beschritten wird. Entstehen nun die Lipidperoxide direkt innerhalb der Lipidmembran, so sind sie aus sterischen Gründen einer Reduktion durch das GSH-Peroxydase-System nur in sehr begrenztem Aus$\mathrm{maß}$ zugänglich, obwohl in vitro Lipidperoxide durch GSH-Peroxydase reduzierbar sind. Die Annahme einer bevorzugt primären Bildung der Lipidperoxide in der Membran würde erklären, weshalb ein Einfluß des GSH-Spiegels auf die Lipidperoxydation in den Untersuchungen von TSEN und Collier (19), JACOB und Lux (16), MENGEL und KanN (17) und in unseren Experimenten nicht feststellbat ist. In diesem Fall wäre der Prozeß effektiver durch eine Tokopherolabhängige Radikal-Ketten-Abbruch-Reaktion zu beeinflussen.

Die Frage, ob der primäre Angriffspunkt von Divicin das Glutathion oder die Membran mit ihren ungesättigten Lipiden ist, bleibt somit unentschieden. Es scheint vielmehr unzulässig, die Frage in dieser alter- nativen Form zu stellen. Wir verfügen über keinerlei experimentelle Evidenz, daß beim Glucose-6-phosphatdehydrogenase-Mangel im reifen Erythrocyten wesentliche Stoffwechselfunktionen außer der Glutathionreduktion gestört sind. Aus der Verwandtschaft der klinischen Bilder von Glucose-6-phosphatdehydrogenase-Mangel und GSH-Peroxydase-Mangel könnte geschlossen werden, $\dot{d} a ß$ die entscheidende Folge der verminderten GSSG-Reduktionsłate beim Glucose-6phosphatdehydrogenase-Mangel die Beeinträchtigung des Hydroperoxidabbaus ist (3). Andererseits wird eine bevorzugte Schädigung von Glucose-6-phosphatdehydrogenase-Mangel-Zellen durch Substanzen beobachtet, deren primärer Angriff an der Erythrocytenmembran erwiesen scheint (30). Zur Lösung der aufgezeigten Widersprüche bietet sich folgende Hypothese an: Bei Beeinträchtigung des cytoplasmatischen Peroxydationsschutzes kommt es in der Erythrocytenmembran chronisch zur Peroxydierung ungesättigter Lipide und zur Oxydation von Membran-SH-Gruppen durch primär in der wäßr. Phase akkumulierte Hydroperoxide. $\mathrm{Da}$ beide Membranschädigungen - zumindest in älteren Erythrocyten - nur begrenzt reparabel sind (16), scheint eine Summation leichter oxydativer Noxen bis zu einem kritischen Ausmaß denkbar, das schließlich zur Lyse des Erythrocyten oder zu seiner Elimination in der Milz ausreicht.

Diese Annahme könnte erstens die verkürzte Lebensdauer enzymopenischer Erythrocyten ohne exogene Belastung erklären. Zweitens wird verständlich, daß sich Glucose-6-phosphatdehydrogenase-Mangel-Zellen nicht durch artifizielle Senkung des GSH-Spiegels gesunder Erythrocyten in vitro simulieren lassen (vgl. Diamidversuche). Drittens kann die Hypothese neben dem erhöhten Glucose-6-phosphatdehydrogenase-Gehalt junger Zellpopulationen zur Deutung des Phänomens der Selbstlimitierung hämolytischer Krisen beitragen. Viertens könnte jedweder peroxidliefernde Autoxydationsprozeß, auch wenn er ausschließlich in der Lipidphase abliefe, die vorgeschädigte Membran einer Glucose-6-phosphatdehydrogenase-Mangel-Zelle bevorzugt schädigen. Selbst membranschädigende Agentien, die primär keinerlei Beziehung zum Peroxidstoffwechsel aufweisen (vgl. 1. c. 30,37) müßten die Glucose-6-phosphatdehydrogenase-Mangelzelle härter treffen. Es ergäben sich somit neue Gesichtspunkte, das breite Spektrum hämolysierender Substanzen zu deuten.

\section{Literatur}

1. Carson, P. E., C. L. Flanagan, C. E. Ickes und A: S. Alving, Science 124, 484 (1956). - 2. Carson, P. E. und H. Frischer, Amer. J. Med. 41, 744 (1966). - 3. Flohé, L., Klin. Wschr. 49, 669 (1971). - 4. Mager, J., G. Glaser und A. Razin, Biochem. biophys. Res. Commun. 20, 235 (1965). - 5. Razin, A., A. Hershko, G. Glaser und J. MAger, Ist. J. Med. Sci. 4, 852 (1968). - 6. Brewer, G. J. und R. J. Dern, Amer. J. hum.
Genet. 16, 472 (1964). - 7. LöHr, G. W. und CH. LAUSEKER, Zit. nach G. W. LöHr, Verb. dtsch. Ges. inn. Med. 70, 495 (1964). 8. LöHr, G. W. und H. D. WaLLER, Med. Klin. $57 I I, 1521$ (1962). - 9. Beutler, E., Pharmacol. Rev. 21, 73 (1969). 10. Oort, M., J. A. Loos und H. K. Prins, Vox. Sang. 6, 370 (1961). - 11. Borvin, P. und C. Galand, Nouv. Rev. Franc. Hematol. 5, 707 (1965). - 12. Kosower, N. S., K.-R. SoNG und 


\section{Moderne digitale Meßtechnik rationalisiert}

\section{photometrische Analysen}

\section{[LEVIZ-Doppelstrahl-Photometer DP-digigita]}

Mit dem neuen LEITZ-Doppelstrahl-Photometer DP-digital werden Ihre MTA oder Ihr Laborant die Routinearbeit problemloser bewältigen. Sie können sich ganz auf die Vorbereitung der Analysen konzentrieren-die Durchführung der Analyse übernimmt das Photometer. Und das Ergebnis erscheint in Bruchteilen von Sekunden in großen Leuchtzahlen: 4-ziffrig, exakt, dezimalstellenrichtig und ablesefehlerfrei.
Einige weitere technische Hinweise:

Automatischer Abgleich

Automatische Meßwertbildung

Automatische Umschaltung der Lichtquellen Automatische Verstärkungsregelung Digitale Anzeige von Transmission, Extinktion oder Konzentration Küvettenautomatik für Enzymkinetiken Anschlußmöglichkeiten für Drucker, Schreiber, Tischrechner

Proben- und Analysenautomatik usw.

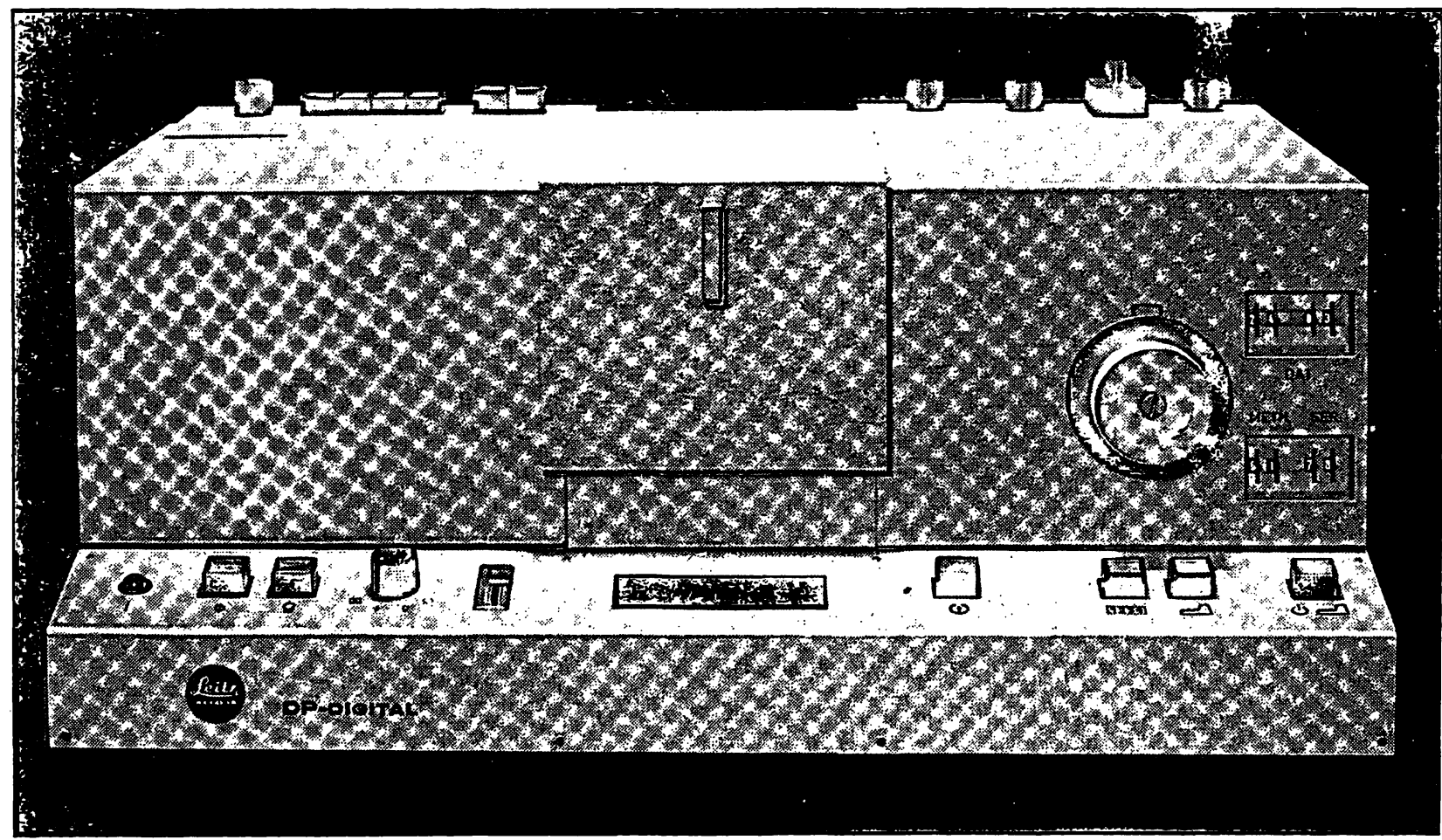

ERNST LEITZ GmbH 6330 Wetzlar Informationsdienst DP 45
Ich bin an Ihrem neuen Doppelstrahl-Photometer DP-digital interessiert und bitte um

den ausführlichen Prospekt [] Name: einen Kostenvoranschlag $\square$ Ort: ( )

Demonstration $\square$ Straße: 
wackelis, ist ein

fumbtionssicheres

und

wartungsfreies

PAiselin- connd

Scholitstelgeratit -

darum solliten Sie

sich wroit uns ín

Verbindunbrg

setzzen, wemn Sie

auf dileserm

Gebies

Probleme habem.

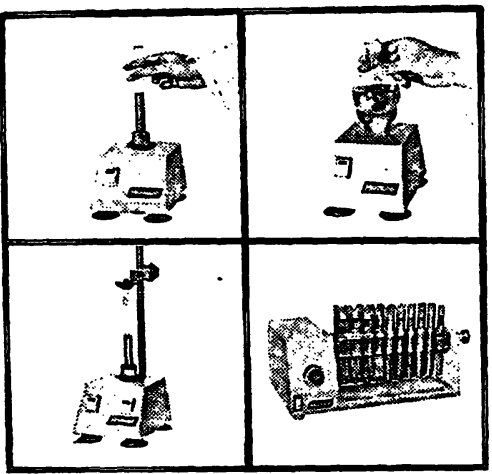

Wir können Ihnen sicher helfen, diese Probleme zu lösen. Unsere Reax-Geräte haben sich in der Praxis bewährt. Sie sind technisch voll ausgereift, außerordentlich leistungsfähig, funktionssicher und außerdem wartungsfrei. Unser Programm umfaßt Einund Mehrfach-Mischgeräte für die verschiedensten Einsatzmöglichkeiten. Bitte fordern Sie Prospektmaterial an.

Unser Fertigungsprogramm umfaßt außerdem: Rührer, Magnetrührer, Verdampfer, Pumpen, Thermostate, Spaltpol- und Kondensatormotoren, Stirnrad- und Schneckengetriebe, stufenlos regelbare Getriebe, Gebläse. Einen ausführlichen Katalog halten wir für Sie bereit.

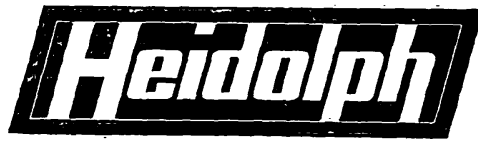

Heidolph-Elektro KG

D 8420 Kelheim/Germany

Tel. 09441 / 75 33, Telex $065415 d$

\section{Buddecke \\ Grundriß der Biochemie}

\author{
für Studierende der Medizin, \\ Zahnmedizin und Naturwissenschaften
}

Von Prof. Dr. Eckhart Buddecke

2. Auflage

Mit mehr als 400 Formeln, Tabellen und Diagrammen

XXXII, 499 Seiten. 1971. Plastik flexibel DM 27,50

Die progressive Zunahme des biochemischen Fachwissens exfordert eine überschanabare und zusammenfassende Darstellung der Biochemie als Hilfsmittel für den Unterricht. Der Grundriß der Biochemie von E. Buddecke gliedert den Wissensstoff in die Kapitel „Stoffe und Stoffwechsel", "Stoffwechselregulation" und „Funktionelle Biochemie der Organe und Gewebe" mit dem Z Ziel, durch knappe Darstellung gesicherter Fakten und gezielte Stoffauswahl dem Leser einerseits eine rasche Information zu bieten, andererseits jedoch auf die vielfältigen Beziehungen und Anwendungsmöglichkeiten der Biochemie zur klinischen Chemie und Molekularpathologie hinzuweisen, um damit dem Studierenden der Medizin und Zahnmedizin sowie der anderen Gebiete der Naturwissenschaften das Verständnis klinischen Fachwissens zu erleichtern.

Stoffe und Stoffwechsel

\section{Inhaltsübersicht}

Chemische Zusammensetzung und Stoffwechsel der Zelle - Der Stoffwechsel als chemische Reaktionsfolge - Enzyme - Coenzyme - Aminosäuren - Nucleinsäuren - Proteine - Glucose und Kohlenhydrate - Lipide - Citratzyklus und biologische Oxydation - Porphyrine - Wasserhaushalt Mineralhaushalt

\section{Stoffwechselregulation}

Selbstregulation durch Rückkopplung - Regulation durch Metabolitkonzentrationen • Enzymkonkurrenz - Hormone

Einführung - Glanduläre Hormone - Gewebshormone

\section{Vitamine}

Definition und Klassifizierung - Vitamine mit Coenzymfunktion - Vitamine obne Coenzymfunktion - Vitaminähnliche Wirkstoffe

\section{Funktionelle Biochemie der Organe und Gewebe}

Biochemie der Zelle - Blut - Leber - Verdauung und Resorption - Niere und Urin - Muskel Nervengewebe $\cdot$ Binde- und Stützgewebe $\cdot$ Wachstum und Abwehr

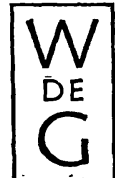


E. M. Kosower, Biochim. biophysica Acta, Amsterdam 192, 23 (1969). - 13. KOSOWER, N. S., E. M. KOSOWER und B. WeRTHEIM, Biochem. biophys. Res. Commun. 37, 593 (1969). - 14. CoHeN, G. und P. Hochstein, Biochemistry, USA 3, 895 (1964). 15. Horwitt, M. K., C. C. Harvey, G. D. Duncan und W. C. Wirson, Amer. J. clin. Nutr. 4, 408 (1956). - 16. JACOB, H. S. und S. E. Lux, Blood 32, 549 (1968). - 17. Menger, С. E. und H. E. KANN jr., J. clin. Invest. 45, 1150 (1966). - 18. Tsen, C. C. und H. B. Collier, Can. J. Biochem. 38, 957 (1960). - 19. Tsen, C. C. und H. B. Collier, Can. J. Biochem. Physiol. 38 , 981 (1960). - 20. Gmelin, R. und G. Hasenmaier, ArzneimittelForschung, Aulendorf 7, 755 (1957). - 21. Davols, J. und D. H. LaNey, J. chem. Soc. $1956 I I, 2124$. - 22. Traube, W. und H. W. Dudley, Chem. Ber. 46, 3839 (1913). - 23. Huls, R., J. chem. Soc. 1956 II, 2033. - 24. CRAwFORD, R. J. und R. RAAP, J. org. Chem. 28, 2419 (1963). - 25. Beutrer, E., O. Duron und B. M. KeLLEY, J.Laborat. clin. Med., St. Louis 61, 882 (1963). - 26.
Hunter, F. E. jr., J. M. Gebickr, P. E. Hoffsten, J. Weinstein und A. Scotr, J. biol. Chemistry 238, 828 (1963). - 27. BARBer, A. A., Rad. Res. Suppl. 3, 33 (1963). - 28. PhrLpot, J. St. L., Rad. Res. Suppl. 3, 55 (1963). - 29. ERICH, C. und H. D. WALLER, Klin. Wschr. 45, 983 (1967). - 30. Mezick, J. A., C. T. SetrieMIRE, G. P. Brierley, K. P. Barefield, W. N. JENSEN und D. G. Cornwelx, Biochim. biophysica Acta, Amsterdam 219, 361 (1970). - 31. Lrtrte, C. und P. J. O'Brien, Biochem. biophys. Res. Commun. 31, 145 (1968). - 32. Christophersen, B. O., Biochim. biophysica Acta, Amsterdam 164, 35 (1968). - 33. Christophersen, B. O., Biochim. biophysica Acta, Amsterdam 176, 463 (1969). - 34. O'BRIEN, R. W., P. D. J. WeItZMAN und J. G. Morris, FEBS letters 10, 343 (1970). - 35. Diskussion zu 1. c. 27. - 36. Farquhar, J. W., Biochim. biophysica Acta, Amsterdam 60, 80 (1962). - 37. Wrrtels, B., Biochim. biophysica Acta, Amsterdam 210, 74 (1970).
Dr. L. Flohé 74 Tübingen Hoppe-Seyler-Str. 1 